\title{
Electronic Properties of Single-Walled Carbon Nanotubes inside Cyclic Supermolecules
}

\author{
Jaakko Akola, ${ }^{*},+,+$ Kari Rytkönen, ${ }^{\ddagger}$ and Matti Manninen \\ Institut für Festkörperforschung, Forschungszentrum Jülich, D-52428 Jülich, Germany, and Nanoscience \\ Center, Department of Physics, University of Jyväskylä, P.O. Box 35, FI-40014 Jyväskylä, Finland
}

Received: August 30, 2005; In Final Form: January 19, 2006

\begin{abstract}
Possible ways for manipulating carbon nanotubes (CNTs) with cyclic supermolecules are studied using density functional theory. Electronic structure calculations with structure optimizations have been performed for the $(4,4)$ and $(8,0)$ single-walled carbon nanotubes (SWNTs) complexed with crown ethers as well as for the $(4,0)$ SWNT with $\beta$-cyclodextrin. A slight polarization of charge in both the nanotube and the supermolecule is observed upon rotaxane complexation, but the interaction is mainly repulsive, and the systems stay $2.8-$ $3.5 \AA$ apart. The supermolecule does not affect the electronic band structure of the nanotube significantly within such a configuration. The situation differs noticeably for chemically cross-linked SWNTs and crown ethers, where a peak arises at the Fermi energy in the density of states. As a result, the band gap of semiconducting CNT $(8,0)(0.5 \mathrm{eV})$ vanishes, and a new conduction channel opens for the metallic CNT $(4,4)$.
\end{abstract}

\section{Introduction}

With the continuing decrease in the size of the components used in electronics and micromechanics, quantum mechanical effects have appeared in their behavior. This has made it difficult to shape nanoscale components arbitrarily with the so-called from-top-to-bottom method, which starts from macroscopic systems and moves gradually toward the atomic limit. A common way to circumvent the difficulties is to mimic nature, where organized structures tend to self-assemble under appropriate conditions. The nanoscale arrangements obtained are often reasonably stable, and they may have similar kinds of structural or electronic properties to those of macroscopic components.

A promising and intensively studied group of self-assembled structures are the carbon nanotubes (CNTs), whose unique mechanical and electronic properties include high structural and chemical stability, high thermal conductivity, electronic properties varying from semiconducting to metallic, and high current carrying capacity. ${ }^{1-4}$ The origins of these properties lie in the one-dimensional structure and nanometer size of CNTs as well as in the strong covalent $\mathrm{sp}^{2}$ bonding within the hexagonal carbon lattice. Recent advances in solution-based chemistry have shown that appropriate processing of CNTs can lead to features normally associated with molecular species, including solubility in organic solvents, solution-based chemical transformations, chromatography, and spectroscopy. ${ }^{5}$ From the technological point of view, single-walled carbon nanotubes (SWNTs) can be used for building molecular sensors and nanoscale devices, and SWNT-based gas sensors have been successfully applied for detecting the molecules $\mathrm{NO}_{2}, \mathrm{NH}_{3}$, and $\mathrm{O}_{2} \cdot{ }^{6,7}$

Rotaxanes and catenanes are other examples of self-assembled structures comprising molecular components that are mechanically interlocked to each other. A rotaxane has a molecular ring threaded on a linear macromolecule with endgroups large enough to prevent the ring from dethreading (bulky stopper),

\footnotetext{
* Author to whom correspondence should be addressed. E-mail: jaakko.akola@phys.jyu.fi.

† Forschungszentrum Jülich

University of Jyväskylä.
}

whereas a catenane is system where two or more molecular rings are attached as in the Olympic rings. The unconventional combination of covalent bonds, robust mechanical links, and noncovalent interactions is responsible for fundamental differences in properties between interlocked macromolecules and covalent polymers. ${ }^{8,9}$

In this paper, we present results for molecular constructions that can be described as polyrotaxanes, namely, crown ether (CE) and $\beta$-cyclodextrin $(\beta$-CD) macrocycles threaded on SWNTs. Previous studies have shown that it is possible to alter both mechanical and electronic properties of nanotubes by modifying their side walls physically and chemically (deformations, defects, B- and N-doping, functional side groups). ${ }^{5,10-15}$ Furthermore, one can use noncovalently attached supermolecules for isolating CNTs and blocking their self-adhesion scheme in polar solvents. ${ }^{16,17}$ Crown ethers and cyclodextrins are interesting molecules in this respect, because they have hydrophobic and hydrophilic parts and tend to attach to organic macromolecules. ${ }^{18,19} \mathrm{We}$ are interested to see whether the conductivity properties of SWNTs can be changed by complexation with a toruslike supermolecule. Large ring cyclodextrins $(\eta$-CD) have been successfully threaded onto SWNTs in water solubilization experiments, enabling their partial separation with respect to the nanotube diameter $(1.2 \mathrm{~nm})$ and determination of a number of CNT types (zigzag, armchair, chiral) with NMR. ${ }^{20}$ For both $\mathrm{CEs}$ and $\beta-\mathrm{CD}$, we note that the addition of the macrocycle does not change the conductivity of the SWNTs, as the intermolecular (polarization) interaction remains relatively small. However, the scheme changes completely if, for instance, some of the CE oxygen atoms are substituted with trifunctional nitrogens that are able to form covalent bonds with the SWNTs.

\section{Simulation Methods}

The electronic structure calculations are performed with the Car-Parrinello molecular dynamics (CPMD) simulation package ${ }^{21}$ which is based on density functional theory. For each atom type, valence electrons are included explicitly, and the electron-ion interaction is described by nonlocal, normconserving, and separable pseudopotentials of the form sug- 
gested by Troullier and Martins. ${ }^{22}$ The generalized gradientcorrected version of exchange-correlation energy functional of Perdew et al. ${ }^{23}$ is adopted, and the plane wave basis set has a kinetic energy cutoff of $70 \mathrm{Ry}$. The program uses periodic boundary conditions, and the electronic band structure calculations are performed with 13 explicit $\mathbf{k}$-points in the reduced Brillouin zone (BZ). To ensure convergence, a finite temperature functional by Alavi et al. ${ }^{24}$ is employed for the Kohn-Sham (KS) single-particle state occupancies with an artificial temperature of $1000 \mathrm{~K}$. The geometries of rotaxane complexes are optimized using the simulated annealing procedure with the $\Gamma$-point only.

Three types of SWNTs are studied in this work: a metallic armchair $(4,4)$ carbon nanotube $(\mathrm{CNT}(4,4))$, a semiconducting zigzag $(8,0)$ nanotube $(\mathrm{CNT}(8,0))$, and a metallic zigzag $(4,0)$ nanotube with a small diameter $(\mathrm{CNT}(4,0))$. The first two SWNTs (diameters 5.51 and $6.36 \AA$, respectively) can be manufactured, whereas the existence of the third one (diameter $3.38 \AA$ ) is still questionable. Some energetic considerations $\mathrm{s}^{25}$ have proposed a lower bound of $4 \AA$ for stable carbon nanotubes, but a recent study by Zhao et al. ${ }^{26}$ provides evidence that $3 \AA$ diameter tubes can be grown inside multiwalled carbon nanotubes (MWNTs) and that the tube in question is most probably an armchair $\mathrm{CNT}(2,2)$.

We report results for three rotaxane systems where the components are (a) $\mathrm{CNT}(8,0)$ and 36-crown-12 ether (CE-12), (b) CNT $(4,4)$ and 30-crown-10 ether (CE-10), and (c) CNT$(4,0)$ and $\beta$-CD. In addition, we have studied the effect of chemical cross-linking in cases $\mathrm{a}$ and $\mathrm{b}$ by replacing four and two ether oxygen atoms by nitrogen (azacrown ether analogues), respectively, and in case $\mathrm{c}$ by forcing two hydroxyl oxygens to form covalent bonds with $\mathrm{CNT}(4,0)$ via hydrogen abstraction. The nanotubes and their rotaxane complexes are modeled by including 3-5 tube unit cells within the simulation box to keep the periodically repeated macrocycles approximately $10 \AA$ apart. The optimized box sizes along the tube axis are $12.32 \AA$ for CNT $(4,4)$ (80 C atoms), $12.82 \AA$ for $\mathrm{CNT}(8,0)$ (96 C atoms), and $16.82 \AA$ for $\mathrm{CNT}(4,0)(64 \mathrm{C}$ atoms). While increasing the computational cost in terms of atoms, valence electrons, and plane waves, this approach enables us to use many fewer k-points than the corresponding unit cell calculations would require. To avoid interactions between rotaxane complexes, the perpendicular box size is adjusted so that the minimum distance between replicas is $8 \AA$ or more.

\section{Results}

The optimized geometries of the CNT $(8,0) @$ CE-12 and CNT$(4,4) @$ CE-10 complexes and their cross-linked forms are shown in Figure 1. Without chemical cross-linking, the CE-12 macrocycle is in a nearly circular form around $\operatorname{CNT}(8,0)$ with the ethyleneoxy units alternating in a zigzag manner (Figure 1a). The average $\mathrm{C}-\mathrm{O}$ distance of $1.433 \AA$ and $\mathrm{C}-\mathrm{O}-\mathrm{C}$ angle of $111.5^{\circ}$ are close to the corresponding gas-phase values $(1.430$ $\AA$ and $111.8^{\circ}$ ), indicating that CE-12 is relatively strain-free. A slight attractive interaction between $\operatorname{CNT}(8,0)$ and $\mathrm{CE}-12$ causes the ether oxygen atoms to approach the tube, and this is reflected in changes of up to $20-30^{\circ}$ in the $\mathrm{O}-\mathrm{C}-\mathrm{C}-\mathrm{O}$ torsional angles. However, the intermolecular $\mathrm{C}-\mathrm{O}$ distances of $3.52 \AA$ are still considerable. The total complexation energy of $-0.12 \mathrm{eV}$ shows that the attraction between $\operatorname{CNT}(8,0)$ and CE-12 oxygens compensates for repulsive terms (steric hindrance, strain). In the case of the CNT $(4,4) @$ CE-10 complex (Figure 1c), a similar conformation is found, but the smaller macrocycle diameter induces additional strain in CE-10, which
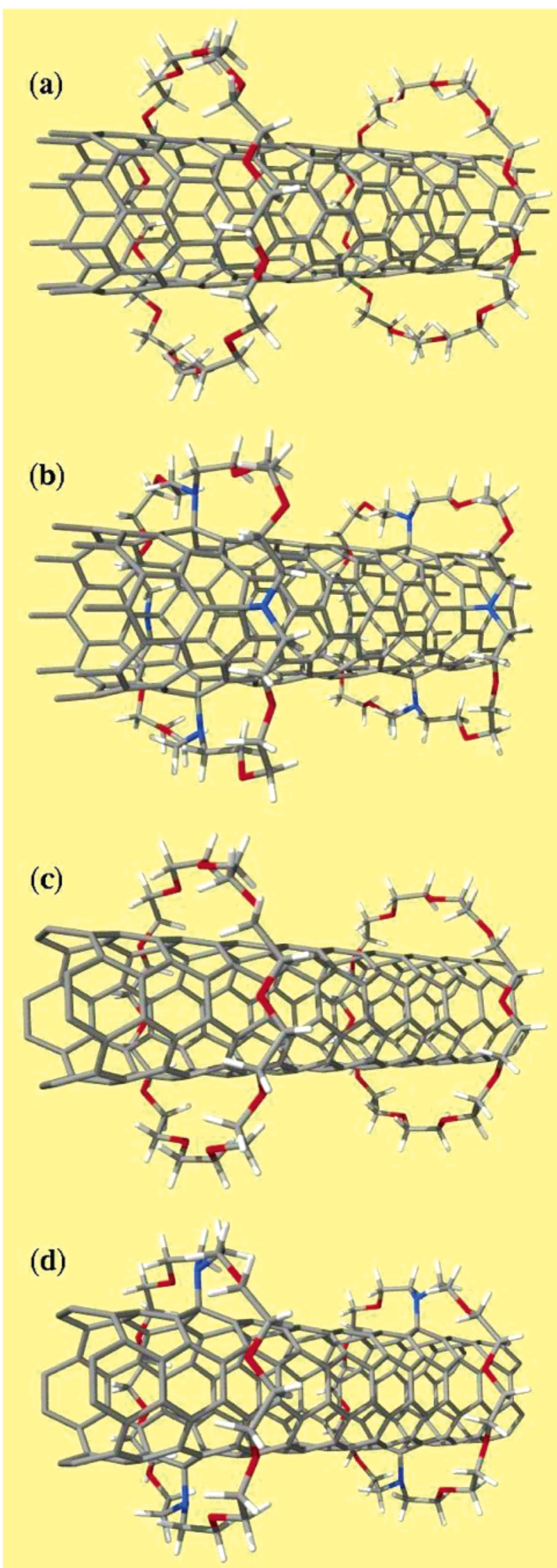

Figure 1. Geometries of $\operatorname{CNT}(8,0)$ and $\operatorname{CNT}(4,4)$ complexes before and after substitution of ether oxygens (red) with trifunctional nitrogens (blue): (a) CNT $(8,0) @ \mathrm{CE}-12$, (b) CNT $(8,0) @ \mathrm{CE}-12 \mathrm{~N} 4$, (c) CNT$(4,4) @ \mathrm{CE}-10$, and (d) CNT $(4,4) @ \mathrm{CE}-10 \mathrm{~N} 2$. The simulated systems are replicated once along the tube axis to show the separation of repeated macrocycles. 
is observed as an elongated $\mathrm{C}-\mathrm{O}$ bond length of $1.446 \AA$ and a positive complexation energy of $1.16 \mathrm{eV}$. The intermolecular $\mathrm{C}-\mathrm{O}$ distances of $2.86 \AA$ are significantly shorter than those for CNT $(8,0) @$ CE-12.

The cross-linked rotaxane system CNT $(8,0) @ \mathrm{CE}-12 \mathrm{~N} 4$ in Figure $1 \mathrm{~b}$ displays four substitutional $\mathrm{N}$ atoms placed symmetrically around $\operatorname{CNT}(8,0)$, resulting in a squarelike overall shape of the macrocycle after formation of covalent $\mathrm{C}-\mathrm{N}$ bonds with the nanotube. The bonding of the corresponding CNT carbons changes from $\mathrm{sp}^{2}$ to $\mathrm{sp}^{3}$, as seen, for example, in the elongated $\mathrm{C}-\mathrm{C}$ distances of $1.52 \AA$ (pure tube $1.42 \AA$ ) and in the $\mathrm{C}-\mathrm{C}-\mathrm{C}$ angles $\left(103^{\circ}\right.$ and $\left.112^{\circ}\right)$, which are close to the tetrahedral value $\left(109.47^{\circ}\right)$. A cross-linked conformation CNT$(4,4) @$ CE-10N2 with two substituted nitrogens on the opposite sides of the macrocycle (Figure 1d) shows the same structural arrangement around the $\mathrm{C}-\mathrm{N}$ bonds, but the overall shape of the macrocycle resembles more the circular CE- 10.

The CNT $(4,0) @ \beta$-CD complex is shown from two perspectives in the charge accumulation/depletion plot in Figure 2. The macrocycle consists of seven glucopyranose rings and resembles initially a truncated cone, and it expands from its narrow end due to steric hindrance effects with the tube. The conformational change is compensated for by attractive interactions between the two components, and the complexation energy is $0.08 \mathrm{eV}$. The circular symmetric hydrogen bond network of the gas-phase conformation is partially broken, and the separation of polarized hydroxyl groups from the tube varies between 2.98 and 3.30 $\AA$. This can also be observed in the nonsymmetrical charge accumulation/depletion lobes that are mainly positioned around the $\beta$-CD narrow edge. A cylindrically averaged charge density difference shows a net accumulation of charge in the hydroxyl oxygens and in the immediate vicinity of $\operatorname{CNT}(4,0)$ (both inside and outside), whereas charge depletion is observed close to the tube axis and in the intermolecular region. There is no significant charge transfer between the two components, and the interaction should be viewed as polarization. The same conclusion can be applied to the CNT $(4,4) @ \mathrm{CE}-10$ and CNT $(8,0) @ \mathrm{CE}-12$ complexes, whereas the charge accumulation/depletion profile is dominated by covalent bond formation in their cross-linked cases.

The cross-linked CNT $(4,0) @ \beta$-CD complex is obtained via hydrogen abstraction in two hydroxyl groups (opposite sides), where the oxygen atoms form covalent $\mathrm{C}-\mathrm{O}$ bonds of $1.45 \AA$ with the tube. As in the previous case, the hybridization of the affected $\mathrm{CNT}(4,0)$ carbons changes from $\mathrm{sp}^{2}$ to $\mathrm{sp}^{3}$, and the tube diameter expands $0.5 \AA$ in the vicinity of contacts. The rest of the macrocycle remains well separated from $\operatorname{CNT}(4,0)$, as in the case without cross-linking.

The electronic band structures of $\mathrm{CNT}(8,0)$ and its complexes with CE-12 and CE-12N4 are shown in Figure 3. As is typical for zigzag nanotubes, the uncovered tube is semiconducting with a band gap of $0.5 \mathrm{eV}$ at the $\Gamma$-point. ${ }^{11,12}$ Evidently, complexation with $\mathrm{CE}-12$ does not perturb the band structure near the Fermi energy, and the only visible effects are the dispersionless bands below $-1.5 \mathrm{eV}$ corresponding to the highest occupied molecular orbitals (HOMOs) of the isolated CE-12. The situation changes considerably when cross-linking is introduced (CE-12N4); the band structure differs from $\operatorname{CNT}(8,0)$ within the interval shown (Figure 3) with only a few familiar features in the conduction band, and a group of four bands is seen at the Fermi energy. Despite the vanishing band gap, there are no conduction channels available, and the system can be described as a semiconductor with a density of states (DOS) shown in Figure 4. The isolated tube has a peaked DOS typical of a one-

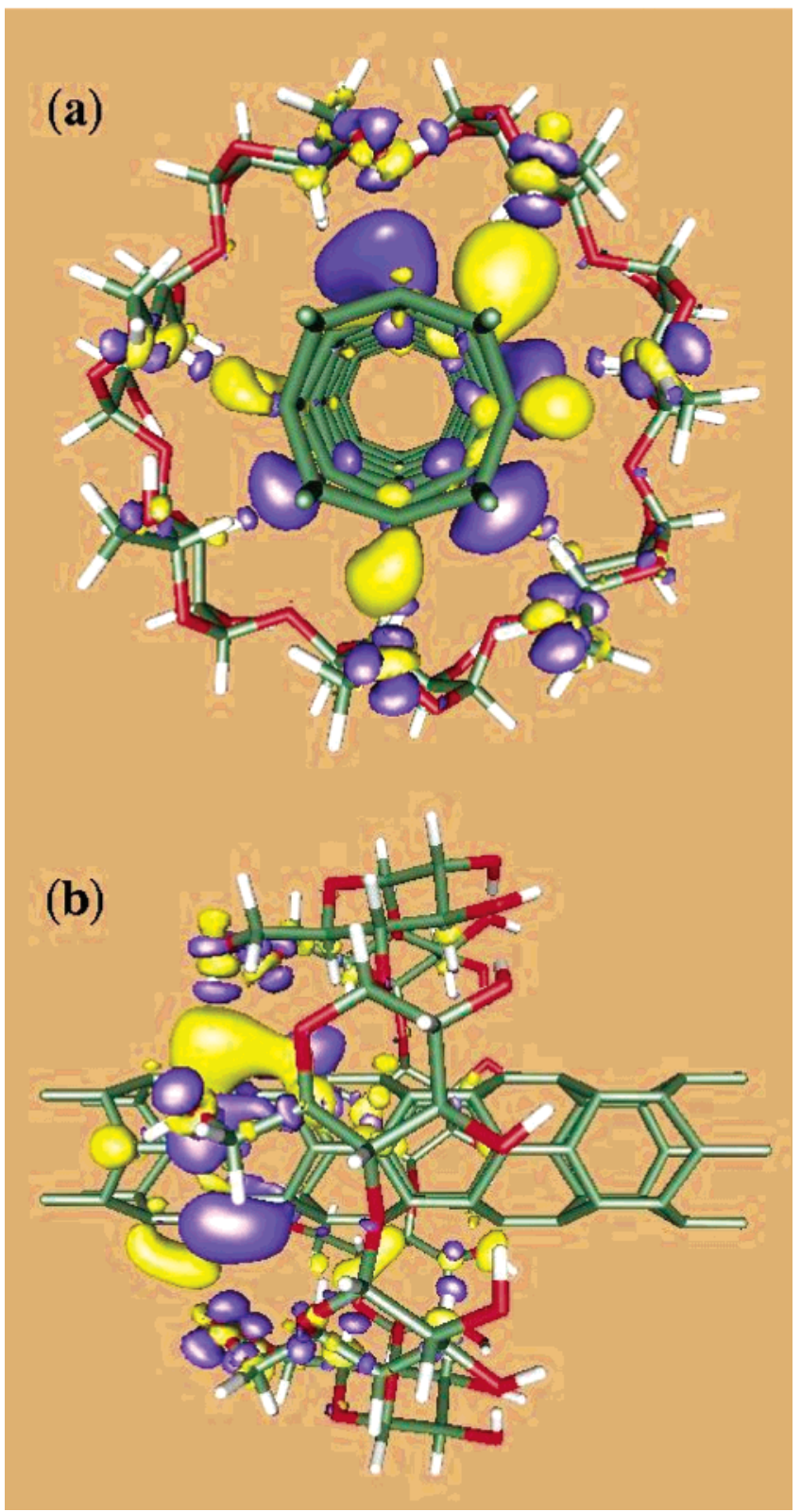

Figure 2. Charge accumulation/depletion plot of the CNT(4,0)@ $\beta$ $\mathrm{CD}$ complex: (a) front view and (b) side view. The isosurface has a value of $0.00045 \mathrm{e}^{\circ} \AA^{3}$ both for accumulation (blue) and depletion (yellow).

dimensional system with van Hove singularities, and the band gap at the Fermi energy is apparent. The cross-linked configuration perturbs the characteristic features of $\operatorname{CNT}(8,0)$, adding new peaks related to the macrocycle. A pronounced peak appears at the Fermi energy (above-mentioned four bands) as the strongest manifestation of covalent bond formation between the two subsystems. However, the DOS of the CNT(8,0)@CE12 complex supports the previous observation that CE-12 does not change the electronic properties of $\mathrm{CNT}(8,0)$.

According to the simple rule based on the nanotube chiral vector, the armchair CNT $(4,4)$ should be conducting. The electronic band structure of CNT $(4,4)$ in Figure 5 confirms this, showing a Fermi energy crossing (conduction channel) near the BZ boundary (X-point), and the corresponding DOS has a finite weight. Our result is in full agreement with the band structure calculated by Rubio et al. ${ }^{27}$ (Note that their Fermi energy crossing appears at a different point in the BZ due to the smaller simulation box of a single unit cell.) The band structure of the 


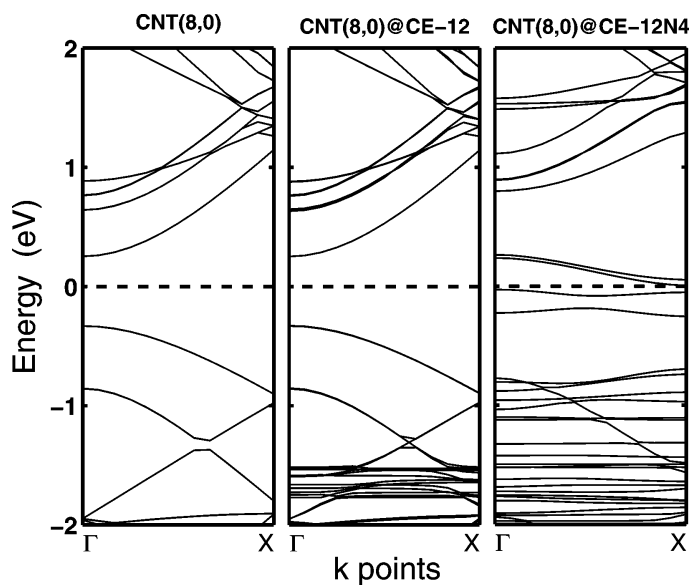

Figure 3. Electronic band structure of $\operatorname{CNT}(8,0)$ with and without CE-12 complexation (cross-linking). The dashed line at $0 \mathrm{eV}$ corresponds to the Fermi energy. The simulation box includes three CNT$(8,0)$ unit cells

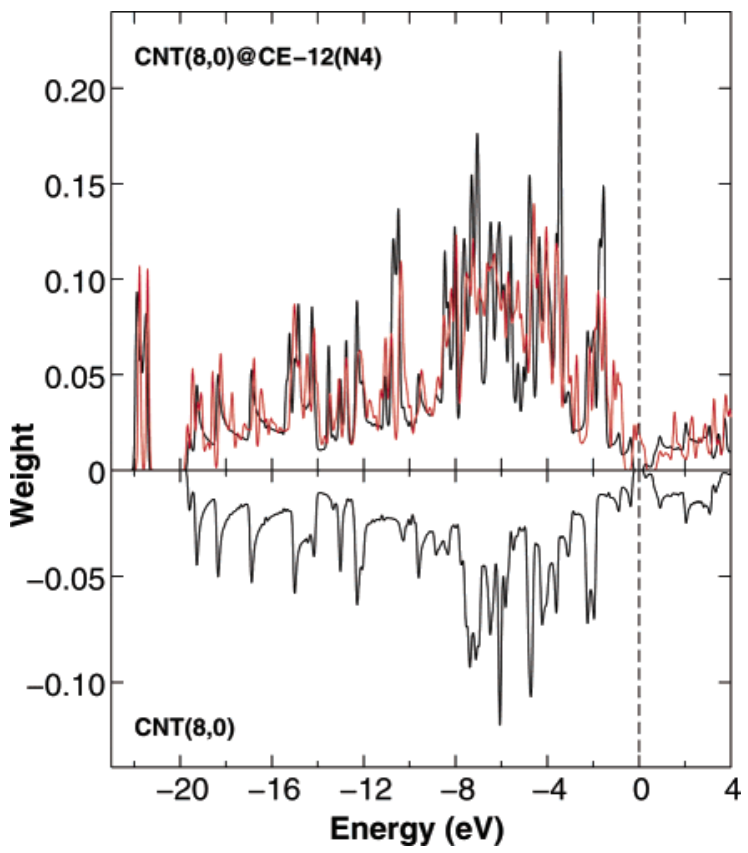

Figure 4. Density of states of the CNT $(8,0) @ \mathrm{CE}-12 \mathrm{~N} 4$ complex in comparison with the pristine tube. The red curve corresponds to the cross-linked complex, and the dashed line at $0 \mathrm{eV}$ marks the Fermi energy.

CNT $(4,4) @$ CE-10 complex is almost identical to that of CNT$(4,4)$, despite the artificially short intermolecular $\mathrm{C}-\mathrm{O}$ distances that enhance polarization effects. However, a cross-linking with CE-10N2 results in significant changes in both valence and conduction bands. In contrast to the pristine tube, there are bands of weak dispersion around the Fermi energy that give rise to a peak in DOS, and a new conduction channel opens, enhancing the metallicity of the system.

Despite belonging to the class of zigzag nanotubes, the band structure of small-diameter CNT(4,0) in Figure 6 implies that the system is metallic with three conduction channels. Previous theoretical considerations have suggested that SWNTs with diameters less than $5 \AA$ are metallic due to the severe tube curvature that causes a strong $\sigma^{*}-\pi^{*}$ hybridization. ${ }^{28}$ Furthermore, it has been proposed that tubes of diameter $4 \AA$ or less should be superconducting. ${ }^{29}$ However, a more detailed analysis by Ito et al. has shown that the main reason that $\mathrm{CNT}(4,0)$ is metallic is the strong $\mathrm{C}-\mathrm{C}$ bond alternation (1.375 and 1.468

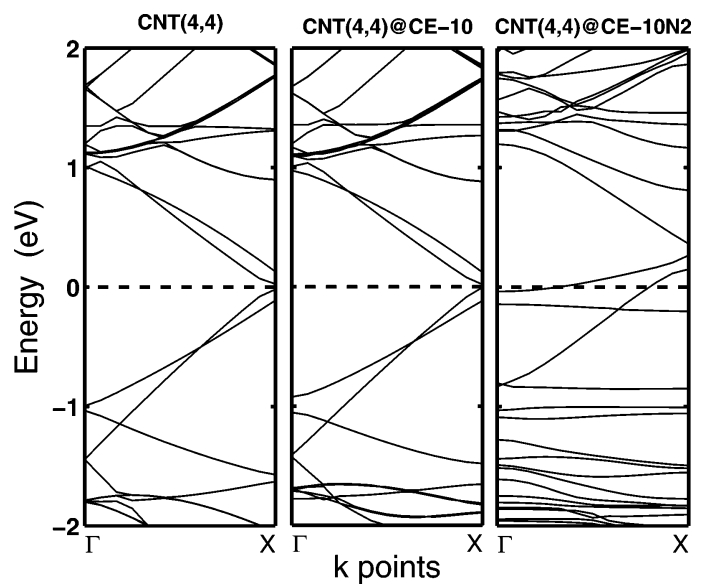

Figure 5. Electronic band structure of $\operatorname{CNT}(4,4)$ with and without CE-10 complexation (cross-linking). The dashed line at $0 \mathrm{eV}$ corresponds to the Fermi energy. The simulation box includes five CNT$(4,4)$ unit cells.

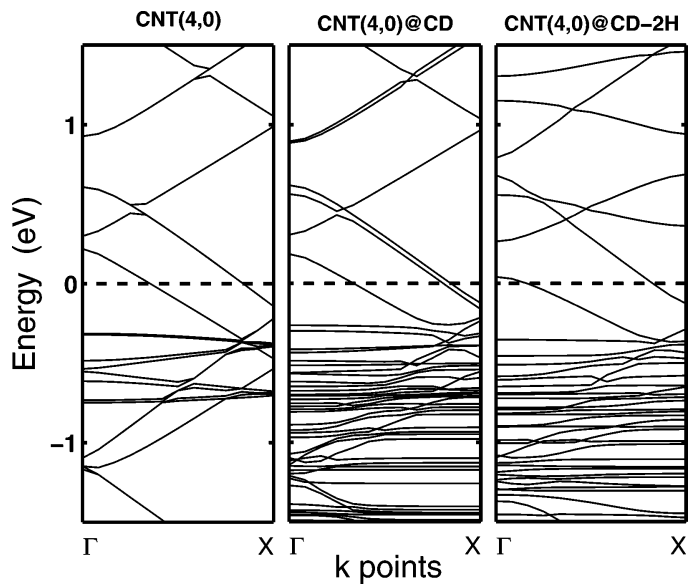

Figure 6. Electronic band structure of $\mathrm{CNT}(4,0)$ with and without $\beta$-CD complexation (cross-linking). The dashed line at $0 \mathrm{eV}$ corresponds to Fermi energy. The simulation box includes four CNT $(4,0)$ unit cells.

$\AA$ ) that lowers (stabilizes) the first unoccupied $\pi^{*}$-band so that it crosses with the highest occupied $\pi$-bands. ${ }^{30}$ Our values for the $\mathrm{C}-\mathrm{C}$ bonds are 1.386 and $1.477 \AA$, and the corresponding bond angles are $119.0^{\circ}$ and $107.9^{\circ}$.

In analogy to larger nanotubes discussed above, a noncovalent complexation of $\mathrm{CNT}(4,0)$ with $\beta$-CD does not change the characteristic bands of the nanotube, whereas the cross-linking with two hydroxyl oxygens has a clear effect. However, the cross-linking suppresses metallicity, and there are only two conduction channels available. The same effect is visible in the corresponding DOS as a smaller weight at the Fermi energy. The probable reason is the elongation of $\mathrm{C}-\mathrm{C}$ bonds at the cross-linking sites $(1.50-1.55 \AA$ ), which breaks the symmetric bond alternation pattern that causes the metallicity of CNT$(4,0)$. The tube curvature is exceptional, and we make no predictions concerning the band structure of larger SWNTs cross-linked with cyclodextrin supermolecules.

\section{Conclusions}

We have studied the possibility of threading SWNTs inside cyclic supermolecules. The obtained polyrotaxane systems are simulated both with and without cross-linking. SWNTs maintain their electronic properties upon noncovalent complexation, whereas cross-linking introduces new features in the electronic band structure. The results obtained should be valuable for future 
work in nanodevices because they show that (a) it is possible to form polyrotaxane systems from SWNTs and cyclic supermolecules where the electronic properties of the SWNT are preserved and (b) chemical cross-linking with macrocycles can tune the conductivity of the SWNT significantly. The first observation could be useful in solution chemistry, where the controlled separation of hydrophobic CNTs in polar solvents (e.g., water) poses a formidable challenge. Cyclodextrins are particularly well-known for their ability to host hydrophobic guest molecules, and the first successful experiments for selforganized CNT-polyrotaxanes have been reported. ${ }^{20}$ The novelty of this approach is that the nanotube diameter must fit the macrocycle, leading to structural selectivity. The latter finding of chemical cross-linking could have an impact in nanoelectronics, where SWNTs are regarded as promising components. Previously, it has been reported that functional $\mathrm{COOH}$ and $\mathrm{NH}_{2}$ side groups alter the SWNT band structure considerably. ${ }^{15}$ We suggest that it should be possible to adjust SWNT conductivity by constructing architectures (junctions, joints, etc.) that resemble the cross-linked polyrotaxanes of this study. The conductivity change suggests also that properly activated CNTrotaxanes could be used as chemical sensors, where the presence of the macrocycle enables a more specific binding site for the target molecule than in the case of a pristine nanotube.

Acknowledgment. Financial support from the Academy of Finland and from the European Community project ULTRA1D (NMP4-CT-2003-505457) is acknowledged. The calculations were performed on IBM-SP4 computers at the John von Neumann Institute for Computing, Forschungszentrum Jülich, Germany, and at the Center for Scientific Computing, Espoo, Finland. We thank R. O. Jones for valuable discussions and a critical reading of the manuscript.

\section{References and Notes}

(1) Iijima, S. Physica B 2002, 323, 1.

(2) Ebbesen, T. W. Phys. Today 1996, 49, 26

(3) Saito, R.; Dresselhaus, G.; Dresselhaus, M. S. Physical Properties of Carbon Nanotubes; Imperial College Press: Singapore, 2004.

(4) Dai, H. Surf. Sci. 2002, 500, 218.

(5) Niyogi, S.; Hamon, M. A.; Hu, H.; Zhao, B.; Bhowmik, P.; Sen, R.; Itkis, M. E.; Haddon, R. C. Acc. Chem. Res. 2002, 35, 1105.
(6) Kong, J.; Franklin, N. R.; Zhou, C.; Chapline, M. G.; Peng, S.; Cho, K.; Dai, H. Science 2000, 287, 622.

(7) Collins, P. G.; Bradley, K.; Ishigami, M.; Zettl, A. Science 2000, 287, 1801.

(8) Raymo, F. M.; Stoddart, J. F. Chem. Rev. 1999, 99, 1643.

(9) Nepogodiev, S. A.; Stoddardt, J. F. Chem. Rev. 1998, 98, 1959.

(10) Terrones, M.; Jorio, A.; Endo, M.; Rao, A. M.; Kim, Y. A.; Hayashi, T.; Terrones, H.; Charlier, J.-C.; Dresselhaus, G.; Dresselhaus, M. S. Mater. Today 2004, 7, 30 .

(11) Fagan, S. B.; Souza Filho, A. G.; Lima, J. O. G.; Mendes Filho, J.; Ferreira, O. P.; Mazali, I. O.; Alves, O. L.; Dresselhaus, M. S. Nano Lett. 2004, 4, 1285.

(12) da Silva, L. B.; Fagan, S. B.; Mota, R. Nano Lett. 2004, 4, 65.

(13) Czerw, R.; Terrones, M.; Charlier, J.-C., Blase, X.; Foley, B.; Kamalakaran, R.; Grobert, N.; Terrones, H.; Tekleab, D.; Ajayan, P. M.; Blau, W.; Rühle, M.; Carroll, D. L. Nano Lett. 2001, 1, 457.

(14) Peng, S.; Cho, K. Nano Lett. 2003, 3, 513.

(15) Zhao, J.; Park, H.; Han, J.; Lu, J. P. J. Phys. Chem. B 2004, 108, 4227.

(16) Zheng, J.; Jagota A.; Strano, M. S.; Santos, A. P.; Barone, P.; Chou, S. G.; Diner, B. A.; Dresselhaus, M. S.; Mclean, R. S.; Onoa, G. B.; Samsonidze, G. G.; Semke, E. D.; Usrey, M.; Walls, D. J. Science 2003, 302, 1545.

(17) Chen, J.; Dyer, M. J.; Yu, M.-F. J. Am. Chem. Soc. 2001, 123, 6201

(18) Gokel, G. W.; Leevy, W. M.; Weber, M. E. Chem. Rev. 2004, 104, 2723.

(19) Del Valle, E. M. M. Process Biochem. 2004, 39, 1033.

(20) Dodziuk, H.; Ejchart, A.; Anczewski, W.; Ueda, H; Krinichnaya, E.; Dolgonos, G.; Kutner, W. Chem. Commun. 2003, 986.

(21) Hutter, J., et al. CPMD Program, version 3.7; Max-Planck-Institut für Festkörperforschung and IBM Research: 1990-2004. http://www.cpmd.org.

(22) Troullier, N.; Martins, J. L. Phys. Rev. B 1991, 43, 1993.

(23) Perdew, J. P.; Burke, K.; Ernzerhof, M. Phys. Rev. Lett. 1996, 77, 3865 .

(24) Alavi, A.; Kohanoff, J.; Parrinello, M.; Frenkel, D. Phys. Rev. Lett. 1994, 73, 2599.

(25) Sawada, S.; Hamada, N. Solid State Commun. 1992, 83, 917.

(26) Zhao, X.; Liu, Y.; Inoue, S.; Suzuki, T.; Jones, R. O.; Ando, Y. Phys. Rev. Lett. 2004, 92, 125502.

(27) Rubio, A.; Miyamoto, Y.; Blase, X.; Cohen, M. L.; Louie, S. G. Phys. Rev. B 1996, 53, 4023.

(28) Blase, X.; Benedict, L. X.; Shirley, E. L.; Louie, S. G. Phys. Rev. Lett. 1994, 72, 1878 .

(29) Tang, Z. K.; Zhang, L.; Wang, N.; Zhang, X. X.; Wen, G. H.; Li, G. D.; Wang, J. N.; Chan, C. T.; Sheng, P. Science 2001, 292, 2462.

(30) Ito, A.; Natsume, Y.; Ohmori, S.; Tanaka, K. Nano Lett. 2002, 2, 629. 